\title{
Effects of Beta-glucan from Coriolus versicolor on Scavenger Receptor B1 Expression and their Involvement of Dectin-1 and Casein Kinase 2
}

\author{
Taeseong Kim, Ye-Jin Kim and Eun-Hwa Sohn* \\ Department of Herbal Medicine Resource, Kangwon National University, Samcheok 245-710, Korea
}

\begin{abstract}
The mushroom Coriolusversicolor contains biologically active polysaccharides, most of which belong to the $\beta$ glucan group. Diverse physicochemical properties, due to different sources and isolated types of $\beta$-glucans, may induce distinct biological activities. Here, we examined the effects of $\beta$-glucan from Coriolusversicolor (CVG) on the scavenger receptor B1 (SR-B1) expression and the role of SR-B1 in CVG-induced phagocytosis regulation by using SR-B1-specific shRNA transfected cells. We also examined whether Dectin-1 and CK2 are involved in SR-B1 expression in CVG-treated cells. Our study results showed that CVG increased the SR-B1 expression via Dectin-1 and CK2 in macrophages. However, the inhibition of SR-B1 expression by shRNA did not completely eliminate the effect of CVG on the increase of phagocytosis suggesting that SR-B1 is not essential for CVG-stimulated phagocytosis. This study will contribute to identify CVG's mechanism of action and its use in the development of functional foods.
\end{abstract}

Key words - Coriolus versicolor, CK2, Dectin-1, $\beta$-Glucan, Macrophage, SR-B1

\section{Introduction}

Coriolus versicolor (known as "Yun Zhi"), a mushroom fungus of the Basidiomycetes family, has been used as a dietary supplement for surgery, chemotherapy, radiation therapy, and rehabilitation (Kidd, 2000; Ho et al., 2005). These mushrooms contain biologically active polysaccharides, most of which belong to the $\beta$-glucans which are naturally occurring $(1 \rightarrow 3)$-b-D-linked polymer glucoses that are found in the cell walls of certain pathogenic bacteria, fungi, mushrooms, algae, and cereal grains (Williams et al., 1992; Muller et al., 1996). Many studies have demonstrated that $\beta$-glucans, either in the form of particulate or soluble, have stimulating effects on the innate immune cells, including macrophages, neutrophils, and natural killer cells, and on the production of cytokines (Ross, 2000; Itoh et al., 1990). Different sources and types of $\beta$-glucans result in diverse physicochemical properties, such as solubility, primary structure, molecular weight, and branching. Interestingly, these variables in the $\beta$-glucan group can induce distinct biological activities, depending on their origins such as antitumor effects and anti-infective properties against

*Corresponding author. E-mail : ehson@kangwon.ac.kr bacterial, viral, fungal, and protozoa infections (Ross, 2000; Itoh et al., 1990).

Phagocytosis plays a critical role in innate immunity by facilitating the removal and killing of pathogens and by priming the adaptive immune response (Janeway and Medzhitov, 2002). The phagocytic process is initiated by the cross-linking of so-called pattern recognition receptors (PRRs), an array of dedicated surface receptors that are capable of innately recognizing non-self structures, such as pathogen-associated molecular patterns (PAMPs). Thus, $\beta$-glucans probably act like PAMPs, and subsequent recognition by appropriate cell surface receptors, such as PRRs, initiates immune responses (Janeway and Medzhitov, 2002). Until now, identified $\beta$-glucan receptor candidates acting as PRRs include Dectin-1, scavenger receptors (SRs), complement receptor 3 (CR3; CD11b/CD18), lactosylceramide (LacCer), and Toll-like receptors (TLRs) (Brown et al., 2002). Among these, Dectin-1 has emerged as the major receptor mediating $\beta$-glucan activity in leukocytes, especially macrophages. Activated macrophages can engulf pathogens through phagocytosis and digest them with lysosomal enzymes. In this process, Dectin-1 plays an important role in fungal recognition by macrophages (Brown et al., 2002; Gantner et al., 2003). 
Scavenger receptors are expressed on various cell types and cell lines, such as myeloid cells (macrophages and dendritic cells) and certain endothelial cells. Scavenger receptors were considered to be involved in anti-fungal defense. Dushkin et al. (1996) and Vereschagin et al. (1998) reported that carboxymethylated glucans were bound to mouse peritoneal macrophages via SRs and other reports suggested that receptors with SR features might be involved in direct (opsonin-independent) recognition of major constituents of fungal cell walls (Hollifield et al., 2007).

Despite the significant therapeutic implications of $\beta$ glucans on phagocytic activities against infectious disease, the molecular mechanisms that mediate these effects are not clear. Our previous studies showed comparable significant effects on phagocytosis and NO production in RAW264.7 macrophages by $\beta$-glucans from yeast, bacteria, and mushrooms, including $C$. versicolor, in vitro. Among them, $\beta$-glucans from $C$. versicolor $(\mathrm{CVG})$ showed a highly significant effect on phagocytosis (Jang et al., 2009; Jang et al., 2010). Thus, we further studied the involvement of scavenger receptor class B1 (SR-B1) expression and its signaling in CVG-stimulated phagocytosis.

\section{Materials and Methods}

\section{Preparation of $\beta$-glucans from mushrooms}

Mushroom $\beta$-glucans isolated from $C$. versicolor were used. The process of isolating and purifying a water-soluble glucan from $C$. versicolor was achieved by hot water extraction, filtration, solvent precipitation, dialysis, and freeze-drying. Acidic fractions of the polysaccharide were separated from crude polysaccharides by DEAE-cellulose anion exchange chromatography at $0.7 \mathrm{M} \mathrm{NaCl}$. The molecular weight of the proteoheteroglycan after Sepharose CL-4B gel filtration chromatography was approximately $750 \mathrm{kDa}$. These products have been shown to possess an $85 \%$ purity level.

\section{Reagents}

Unless stated otherwise, all chemicals were purchased from the Sigma Chemical Co. (St. Louis, MO). The RPMI 1640 medium and fetal bovine serum (FBS) were purchased from GIBCO (Grand Island, NY). All of the cell culture reagents and $\beta$-glucans were assayed for any endotoxin contamination using the Limulus lysate test (E-Toxate, Sigma), and the endotoxin levels were found to be $<10 \mathrm{pg} / \mathrm{ml}$. Antibody against SR-B1 was purchased from Abcam Inc. (Cambridge, MA). Antibody for $\beta$-actin was from Cell signaling (Beverly, MA). Laminarin and 2-Dimethylamino4,5,6,7-tetrabromo-1H-benzimidazole (DMAT) were obtained from Sigma Chemical Co. (St Louis, MO).

\section{Cell culture}

The RAW264.7 mouse monocyte/macrophage cell line was maintained in RPMI1640 medium supplemented with $10 \%$ FBS and $2 \%$ penicillin/streptomycin, and was incubated at $37^{\circ} \mathrm{C}$ in $5 \% \mathrm{CO}_{2}$.

\section{Reverse transcription-polymerase chain reaction analysis}

Total RNA was extracted from RAW264.7 cells treated with $\operatorname{CVG}(1,10$, and $100 \mu \mathrm{g} / \mathrm{ml})$ for $16 \mathrm{~h}$ using Trizol. The total amount of harvested RNA was measured using a UV/Vis spectrophotometer at $260 \mathrm{~nm}$. One microgram of the total RNA was reverse-transcribed using SuperScript II (Invitrogen Life Technologies, Carlsbad, CA, USA). Polymerase chain reaction (PCR) was then performed in a final volume of $20 \mu \mathrm{l}$ containing $1 \mu \mathrm{l}$ of template, $0.25 \mu \mathrm{l}$ of Taq DNA polymerase (Takara, Seoul, Korea), and $20 \mathrm{nmol}$ of each primer. The PCR cycler was programmed for 20 cycles for GAPSH and SR-B1. Each cycle was held at $94^{\circ} \mathrm{C}$ for $30 \mathrm{~s}, 60^{\circ} \mathrm{C}$ for $30 \mathrm{~s}$, and $72^{\circ} \mathrm{C}$ for $60 \mathrm{~s}$. The PCR products were separated on a 1.2\% agarose/ ethidium bromide gel, which was then photographed under UV illumination. The single-stranded cDNA was amplified by PCR using specific primers. The sequences of the primers used in this study were SR-B1 forward: 5'-CTCCCAGACATGCT TCCCATA-3'; SR-B1 reverse: 5'-CTCAGCTTCATGGAC CTGCA-3'; GAPDH forward: 5'-CCATGGAGAAGGCTG GGG-3'; GAPDH reverse: 5'-CAAAGTTGTCATGGATGA CC-3'. The quantity of each transcript was calculated as described in the instrument manual and normalized to the amount of GAPDH, a housekeeping gene.

\section{Determination of phagocytosis}

The phagocytic activity was measured using the assay system described previously (Okimura et al., 1986; Choung 
and Sohn, 2011). Cells were incubated with $1 \times 10^{6}$ particle of zymosan and $600 \mu \mathrm{g} / \mu \mathrm{l}$ of nitroblue tetrazolium (NBT). After $1 \mathrm{hr}$ of incubation, plates were centrifuged at $4^{\circ} \mathrm{C}$ to stop an ingestion of zymosan and this was followed by the removal of supernatant by flipping. The optical density of the reduction product of NBT, a purple insoluble formazan, was determined at $540 \mathrm{~nm}$ using a Molecular Devices microplate reader. It was not necessary to solubilize formazan before the determination of absorbance.

\section{Westem blot analysis}

Cells were harvested and lysed in ice-cold lysis buffer (50 $\mathrm{mM}$ Tris- $\mathrm{HCl}$ [pH 7.4], $150 \mathrm{mM} \mathrm{NaCl}, 1 \mathrm{mM}$ EDTA, $0.5 \%$ $[\mathrm{v} / \mathrm{v}] \mathrm{NP}-40,0.1 \%[\mathrm{w} / \mathrm{v}]$ SDS) containing protease inhibitor cocktail (Roche Diagnostics Corp., Indianapolis, IN, UAA) for $1 \mathrm{~h}$. Protein concentrations were measured using the DC Protein Assay (Bio-Rad Laboratories, Hercules, CA, USA) with bovine serum albumin as the standard. Twenty micrograms of each sample was electrophoresed on $10 \%$ sodium dodecylsulfate polyacrylamide gel electrophoresis gels and transferred to Hybond-ECL nitrocellulose membranes (Amersham Biosciences, Piscataway, NJ, USA). The membranes were blocked with $5 \%$ skim milk in Tris-buffered saline/non-fat Tween for $1 \mathrm{~h}$. The membranes were probed with the appropriate antibodies. They were then washed with Tris-buffered saline/Tween 20 once for $15 \mathrm{~min}$ and three times for $5 \mathrm{~min}$ and incubated with secondary HRP-conjugated antibody for $1 \mathrm{~h}$. The blots were developed using an enhanced chemoluminescence kit (Amersham). In all immunoblotting experiments, the blots were reprobed with an antibody against $\beta$-actin which were used as protein loading controls.

\section{Cell transfection and RNA interference}

Transient transfection was performed using psuperNeoGFP (Oligoengine, Seattle, WA). According to the manufacturer's instructions, RAW264.7 cells $\left(1 \times 10^{6}\right.$ cells $\left./ \mathrm{ml}\right)$ were seeded in 6-well plates $24 \mathrm{~h}$ before transfection. Appropriate mixtures of Lipofectamine (Invitrogen, Carlsbad, CA) with plasmid containing shRNA sequence targeting SR-B1 were added to each well containing DMEM. The shRNA sequence used against murine SR-B1 was 5'-GCTCGGCGTTATCATGATT-3'. Non-targeting shRNA served as a control DNA sequence with no matches upon BLAST search. After $48 \mathrm{~h}$ of incubation, the medium was removed and cells were harvested for experiments.

\section{Statistical analyses}

Each experiment was repeated three or four times, and the results of a representative experiment are shown. The results are expressed as the means \pm SEM and were compared using Student's $t$-test. A statistical probability of $p<0.05$ was considered significant ( $\# p<0.05$ and $* p<0.05$ ).

\section{Results and Discussion}

In this study, we demonstrated that $\beta$-glucans from $C$. versicolor (CVG) increased the SR-B1 expression via Dectin-1 signaling through the activation of casein kinase 2 (CK2). Additionally, we showed that inhibition of SR-B1 expression by shRNA did not completely remove the effect of CVG on phagocytosis increase, suggesting that SR-B1 is not essential for CVG-induced phagocytoic process in macrophages. We suggested in the previous study that CVG showed stronger phagocytic activity than the ones from microorganism and seaweeds (Jang et al., 2009) and explained that its phagocytosis was promoted through Dectin-1 signaling.

SR-B1 is a multifunctional receptor capable of binding a wide array of native and modified lipoproteins (Greaves et al., 1998). Its primary function is promoting the selective uptake of cholesteryl esters from high density lipoprotein (HDL). SR-B1 also mediates the activation of endothelial nitric oxide synthase production by HDL (Yuhannaet al., 2001). However, the exact role of SR-B1 in the phagocytic process needs more study. Therefore, we studied the involvement of SR-B1 expression in CVG-induced phagocytic signals and newly suggested possibility of SR-B1 expression which signals through by Dectin-1 and CK2.

Our results showed that CVG increased SR-B1 mRNA and protein expression levels in a dose-dependent manner (Fig. 1). The discovery of Dectin-1 and the study of its functions have helped to assess many of the previously reported roles of $\beta$-glucan receptors in immunity including the innate recognition of and response to fungal pathogens and $\beta$-glucan-mediated immunomodulation. Though there are other $\beta$-glucan receptors 


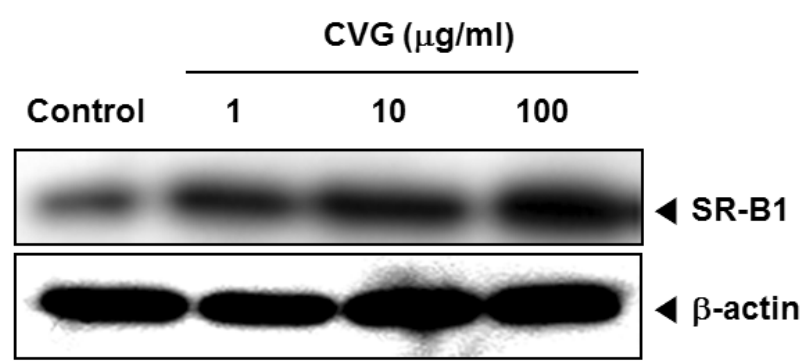

(A)

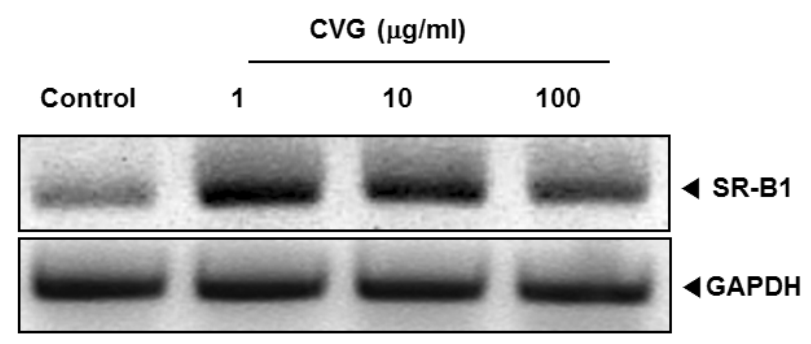

(B)

Fig 1. Effects of $\beta$-glucan from C. versicolor (CVG) on SR-B1 expression in RAW264.7 cells. (A) Cells were treated with various concentrations of CVG for $24 \mathrm{~h}$. The level of SR-B1 expression was determined by western blot assay. (B) Cells were treated with various concentrations of CVG for $16 \mathrm{~h}$. Total RNA was extracted and subjected to reverse transcription polymerase chain reaction analysis.

on leukocytes such as SRs, CR3, LacCer, and TLRs, the use of specific antagonists and blocking monoclonal antibodies has clearly shown that Dectin-1 is the main receptor which mediates immune-modulating activity in leukocytes, particularly macrophages and dendritic cells (Willment et al., 2005; Brown et al., 2002). The importance of Dectin-1 in the activation of innate immune responses of macrophages is also supported by a report which proved the knockout of the Dectin-1 gene and consequent abolition of all macrophage-mediated responses (Taylor et al., 2007).

Addition of laminarin, a well-established Dectin-1 inhibitor, can significantly block Dectin-1 receptors and inhibit surface expression of Dectin-1 (Brown et al., 2002; Gantner et al., 2003). Thus, we used laminarin to examine whether SR-B1 expression was affected by major $\beta$-glucan receptor Dectin-1 signaling in CVG-induced macrophages. As shown in Fig. 2, CVG abrogated the increase of SR-B1 expression in the presence of laminarin.

CK2, a ubiquitous serine/threonine kinase, is one of the

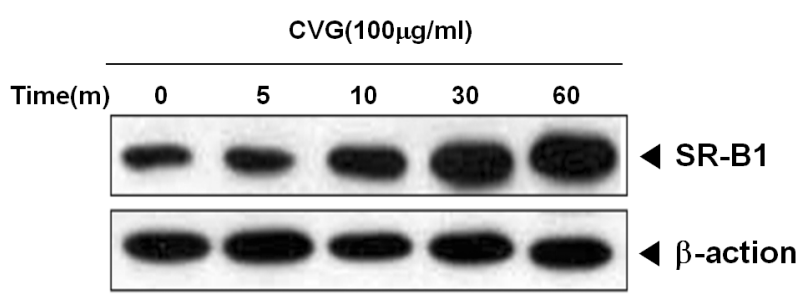

(A)

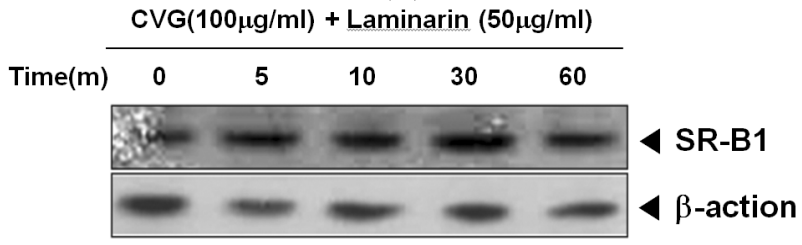

(B)

Fig. 2. Inhibitory effects of laminarin, Dectin-1 specific inhibitor, on SR-B1 expression by $\beta$-glucans from C. versicolor (CVG) in RAW264.7 cells. (A) Cells were treated with CVG for various times. (B) Cells were treated with CVG in the presence or absence of laminarin $(50 \mu \mathrm{g} / \mathrm{ml})$ for various times. The level of SR-B1 expression was determined by western blot assay.

most highly conserved proteins in eukaryotic cells. A lot of evidence suggests that CK2 plays a role in cell survival signals and controls a wide range of processes, including the regulation of cell cycle, apoptosis, transformation, and circadian rhythm (Duncan and Litchfield, 2008). Overexpression of CK2 has been associated with the development of various types of tumors, including mammary gland, prostate, lung, and kidney cancers. Elevated activities of CK2, whether from the subunits alone or of the heterotetrameric holoenzyme, may reflect the pleiotropy of this kinase, which is supported by the fact that more than 300 substrates have been identified so far (Unger et al., 2004). In normal cells, the level of CK2 appears to be tightly regulated and cells resist the change in their intrinsic CK2 level. Disruption of CK2 by treatment of cells with antisense CK2 resulted in the induction of apoptosis in cancer cells and in this regard, CK2 has been considered as an important factor in cancer treatment (Unger et al., 2004; Duncan and Litchfield, 2008). Here, we examined the possibility of CK2-regulated SR-B1 expression in CVG-stimulated macrophages. To determine whether CK2 is involved in SR-B1 expression of CVG, macrophages were treated with $\mathrm{CVG}$ in the presence or absence of CK2 inhibitor, DMAT $(2 \mu \mathrm{M})$. The increase of SR-B1 expression has decreased in the presence 
of DMAT, implicating that CK2 signaling is involved in the SR-B1 expression in CVG-stimulated macrophages (Fig. 3).

Mannose and FcR receptors involved in phagocytosis of macrophage have the so-called "receptor recovery effect", the ability to recycle or newly synthesize the proper receptors (Goodridge et al., 2011). Even though the main function of SR family is known to uptake and clean a broad range of microorganism and modified molecules such as phagocytosis, there has been no clear understanding of the correlation between macrophage activity and scavenger receptor expression (Peiser et al., 2002). Based on the assumption that CVGstimulated phagocytosis may happen by increasing the expression

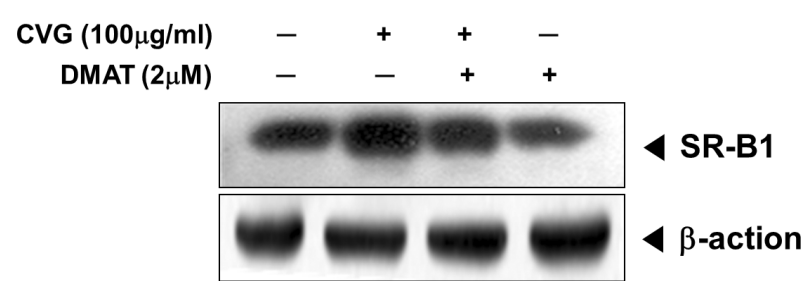

Fig. 3. CK2-dependent increase of SR-B1 expression by $\beta$ -glucan from C. versicolor (CVG) in RAW264.7 cells. Cells were treated with CVG $(100 \mu \mathrm{g} / \mathrm{ml})$ in the presence or absence of CK2 inhibitor, DMAT $(2 \mu \mathrm{M})$ for $24 \mathrm{hrs}$. The level of SR-B1 expression was determined by western blot assay.

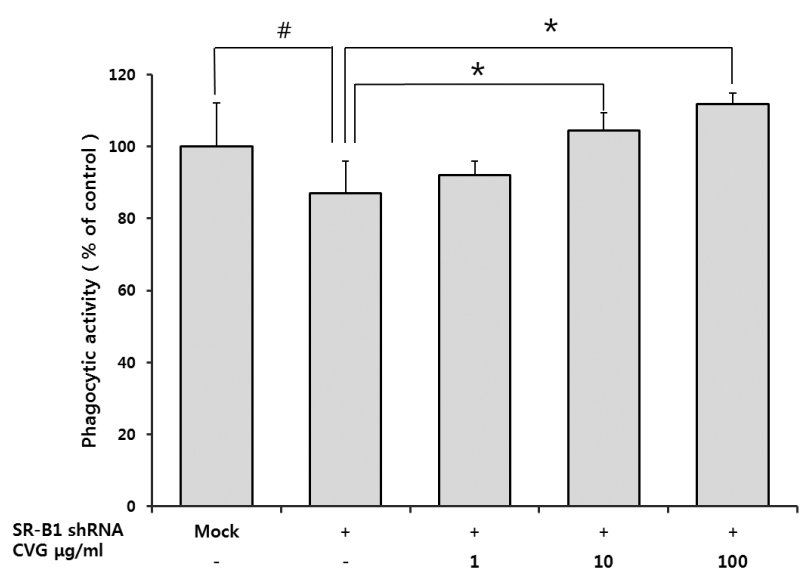

Fig. 4. Involvement of SR-B1 expression in $\beta$-glucan from $C$. versicolor (CVG)-stimulated phagocytosis. RAW264.7 cells were transfected with mock control, non-targeting control or SR-B1-specific shRNA. Cells were treated with CVG and subjected to phagocytic activity assay. The graphs represent the mean of three experiments \pm SEM. ${ }^{\#} \mathrm{P}<0.05$ : significantly different from mock control, ${ }^{*} \mathrm{P}<0.05$ : significantly different from SR-B1 shRNA alone. of SR-B1 receptor, we further studied the involvement of SR-B1 expression in CVG-stimulated phagocytosis using SR-B1-specific shRNA transfected cells. As shown in Fig. 4, CVG treatment has not stop the increase of phagocytosis in SR-B1 shRNA-transfected cells, indicating SR-B1 expression is not involved in CVG-induced phagocytosis.

Collectively, our study demonstrates that the $\beta$-glucan from C. versicolor increases SR-B1 expression via Dectin-1 signaling through the activation of CK2 pathway and the expression of SR-B1 is not essential for activating phagocytosis in CVGtreated macrophages. We believe this study will contribute to identify the action mechanism of $\beta$-glucan from mushroom and its use in the development of functional foods.

\section{Literature Cited}

Brown, G.D., P.R. Taylor, D.M. Reid, J.A. Willment, D.L. Williams, L. Martinez-Pomares, S.Y. Wong and S. Gordon. 2002. Dectin-1 is a major $\beta$-glucan receptor on macrophages. J. Exp. Med. 196:407-412.

Choung, M.G. and E.H. Sohn. 2011. Anti-tumor activity of saponin fraction of Platycodon gradiflourm through immunomodulatory effects associated with NO production in RAW264.7 cells. J. Korean Plant Res. 24(5): 557-563 (in Korean).

Duncan, J.S. and D.W. Litchfield. 2008. Too much of a good thing: the role of protein kinase CK2 in tumorigenesis and prospects for therapeutic inhibition of CK2. Biochim. Biophys. Acta. 1784(1):33-47.

Dushkin, M.I., A.F. Safina, E.I. Vereschagin and Y.S. Schwartz. 1996. Carboxymethylated $\beta$-1,3-glucan inhibits the binding and degradation of acetylated low density lipoproteins in macrophages in vitro and modulates their plasma clearance in vivo. Cell Biochem. Funct. 14:209-217.

Gantner, B.N., R.M. Simmons, S.J. Canavera, S. Akira and D.M. Underhill. 2003. Collaborative induction of inflammatory responses by Dectin-1 and toll-like receptor. J. Exp. Med. 197:1107-1117.

Greaves, D.R., P.J. Gough and S. Gordon. 1998. Recent progress in defining the role of scavenger receptors in lipid transport, atherosclerosis and host defence. Curr. Opin. Lipidol. 9: 425-932.

Goodridge, H.S., C.N. Reyes, C.A. Becker, T.R. Katsumoto, J. Ma, A.J. Wolf, N. Bose, A.S. Chan, A.S. Magee, M.E. Danielson, A. Weiss, J.P. Vasilakos and D.M. Underhill. 
2011. Activation of the innate immune receptor Dectin-1 upon formation of a 'phagocytic synapse'. Nature 472(7344): 471-475.

Ho, C.Y., C.F. Kim, K.N. Leung, K.P. Fung, T.F. Tse, H. Chan and C.B. Lau. 2005. Differential anti-tumor activity of Coriolus versicolor (Yunzhi) extract through p53- and/or Bcl-2-dependent apoptotic pathway in human breast cancer cells. Cancer Biol. Ther. 4:638-644.

Hollifield, M., E.B. Ghanem, W.J.S. de Villiers and B.A. Garvy. 2007. Scavenger receptor A dampens induction of inflammation in response to the fungal pathogen Pneumocystis carinii. Infect. Immun. 75:3999-4005.

Itoh, W., I. Sugawara, S. Kimura, K. Tabata, A. Hirata, T. Kojima, S. Mori and K. Shuimada. 1990. Immunopharmacological study of sulfated schizophyllan (SPG) I. Its action as a mitogen and anti-HIV agent. Int. J. Immunopharmacol. 12:225-233.

Janeway, C.A. and R. Medzhitov. 2002. Innate immune recognition. Annu. Rev. Immunol. 20:197-216.

Jang, S.A., S. Namkoong and E.H. Sohn. 2010. Effects of $\beta$ -glucans from Coriolus versicolor on macrophage functions in young and aged mice J. Korean Plant Res. 23(6):498-502.

Jang, S.A., S. Park, J.D. Lim, C.H. Kang, K.H. Yang, S. Pyo and E.H. Sohn. 2009. The comparative immunomodulatory effects of $\beta \beta$-glucans from yeast, bacteria, and mushroom on the function of macrophages. J. Food Sci. Nutr. 14:102-108.

Kidd, P.M. 2000. The use of mushroom glucans and proteoglycans in cancer treatment. Altern. Med. Rev. 5:4-27.

Muller, A., P.J. Rice, H.E. Ensley, P.S. Coogan, J.H. Kalbfleisch, J.L. Kelley, E.J. Love, C.A. Portera, T. Ha, I.W. Browder and D.L. Williams. 1996. Receptor binding and internalization of water-soluble $(1 \rightarrow 3)-\beta$-D-glucan biologic response modifier in two monocyte/macrophage cell lines. J. Immunol. 156: 3418-3425.

Okimura, T., M. Ogawa and T. Yamauchi. 1986. Stress and immune responses. III. Effect of resistant stress on delayed type hypersensitivity (DTH) response, natural killer (NK) activity and phagocytosis in mice. Jpn. J. Pharmacol. 41: 229-235.

Peiser, L., S. Mukhopadhyay and S. Gordon. 2002. Scavenger receptors in innate immunity. Current Opinion in Immunology 14:123-128.

Ross, G.D. 2000. Regulation of the adhesion versus cytotoxic functions of the Mac-1/CR3/alphaMbeta2-integrin glycoprotein. Crit. Rev. Immunol. 20:197-222.

Taylor, P.R., S.V. Tsoni, J.A. Willment, K.M. Dennehy, M. Rosas, H. Findon, K. Haynes, C. Steele, M. Botto, S. Gordon and G.D. Brown. 2007. Dectin-1 is required for $\beta$-glucan recognition and control of fungal infection. Nat. Immunol. 8:31-38.

Unger, G.M., A.T. Davis, J.W. Slaton and K. Ahmed. 2004. Protein kinase CK2 as regulator of cell survival: implications for cancer therapy. Curr. Cancer Drug Targets 4(1):77-84.

Vereschagin, E.I., A.A. Van Lambalgen, M.I. Dushkin, Y.S. Schwartz, L. Polyakov, A. Heemskerk, E. Huisman, L.G. Thijs and G.C. Van den Bos. 1998. Soluble glucan protects against endotoxin shock in the rat: the role of the scavenger receptor. Shock 9:193-198.

Williams, D.L., H.A. Pretus and I.W. Browder. 1992. Application of aqueous gel permeation chromatography with in-line multi-angle laser light scattering and differential viscometry detectors for the characterization of natural product carbohydrate pharmaceuticals. J. Liq. Chromatogr. 15:2297-2309.

Willment, J.A., A.S.J. Marshall, D.M. Reid, D. L. Williams, S.Y. Wong, S. Gordon and G.D. Brown. 2005. The human $\beta$ -glucan receptor is widely expressed and functionally equivalent to murine Dectin-1 on primary cells. Eur. J. Immunol. 35: 1539-1547.

Yuhanna, I.S., Y. Zhu, B.E. Cox, L.D. Hahner, S. OsborneLawrence, P. Lu, Y.L. Marcel, R.G. Anderson, M.E. Mendelsohn, H.H. Hobbs and P.W. Shaul. 2001. High-density lipoprotein binding to scavenger receptor-B1 activates endothelial nitric oxide synthase. Nat. Med. 7:853-857. 